\title{
2nd European Congress of Andrology
}

\author{
Malmö \\ September 19th-22nd, 2002
}

\section{Thursday, $19^{\text {th }}$ September, 2002}

\section{Postgraduate Course}

Contribution of molecular biological research to clinical andrology

Y chromosome microdeletions (J Gromoll, DE)

Molecular mechanisms in spermatogenesis (JA Grootegoed, NL)

LH receptor knockout (I Huhtaniemi, FI)

Mammalian sex-reversal and intersexuality (D Vaiman, FR)

21-hydroxylase deficiency (W Wedell, SE)

cDNA microarrays - potential role in andrological research ( $\AA$ Borg, SE)

Paediatric and adolescent andrology

Sex differentiation. From genotype to phenotype (J Müller, DK)

Physiological and delayed puberty (P Kumanov, BG)

Androgen resistance in childhood (IA Hughes, UK)

Genetic causes of hypogonadotropic hypogonadism (PMG Bouloux, UK)

Cryptorchidism (J Toppari, FI)

Male precocious puberty (G Saggese, IT)

\section{Main Congress}

Opening Ceremony and Get-together Buffet

\section{Friday, $20^{\text {th }}$ September, 2002}

\section{Enviromental Effects on Male Reproductive Health}

How working place conditions, environmental toxicants and life style affect male reproductive function.

\section{J.P. Bonde}

Environment and male gonadal dysgenesis syndrome. N.E. Skakkebaek

Estrogen hypothesis: where do we stand now? R. Sharpe 


\section{Estrogen receptor and male reproduction}

\section{Cancer and Male Reproductive Function}

The effect of cancer therapy on pituitary-gonadal axis. S.M. Shalet

Sperm DNA damage as a consequence of cancer therapy. I.D. Morris

Testosterone suppression stimulates recovery of spermatogenesis after cancer treatment. M.L. Meistrich

\section{Erectile Dysfunction}

Erectile dysfunction after surgical treatment. V. Mirone

Pathophysiology of erectile dysfunction. F. Montorsi

New trends in therapy of erectile dysfunction. K.E. Andersson

\section{Saturday, $21^{\text {st }}$ September, 2002}

\section{Genetic Analysis of Male Infertility: Safety aspects of ICSI}

$\mathrm{Y}$ chromosomes and male fertility and infertility. C.G. Krausz

CFTR screening before ART M.R. Gazvani

ICSI : a technique too far? H. Tournaye

\section{Andrological aspects of doping in sport. S. Bhasin}

\section{Prostatic Cancer}

PSA and hK2 as markers of prostatic cancer. H. Lilja

Should we screen for prostate cancer? F.C. Hamdy

Hormonal treatment of prostate cancer. F.M.J. Debruyne

Androgen receptor and prostatic cancer invasion. L. Bonaccorsi

\section{Ejaculatory Dysfunction}

Medical treatment of ejaculatory disturbances. A. Kamischke

Vibration and electrostimulation in treatment of ejaculatory dysfunction. J. Sonksen

Sexological approach to ejaculatory dysfunction. E.A. Jannini

Recent development of hormonal male contraception. F. Wu. 


\section{Androgen Receptor}

Androgen receptor and male infertility. O. Hiort

Androgen receptor gene - structural changes in relation to the function of the receptor. A.C. Cato

Polymorphism of androgen receptor gene maleness. E. Nieschlag

Endocrine and urologic changes in normally aging men: Results from Massachusetts Male Aging Study. J.B. McKinlay

Androgen Replacement Therapy and ageing male. I. Huhtaniemi, J.B. McKinlay, J.E. Morley, F. Wu

INFORMATIONS

www.kir.mas.lu.se/eca2002

info@malmo-congress.com

Fax : +46 40258559 


\section{CONGRÈS FRANCOPHONES}

\section{FORUM MÉDITERRANÉEN D'ANDROLOGIE 2002 DE L'ASSOCIATION MÉDITERRANÉENNE D'ANDROLOGIE}

7-8 Juin 2002

\section{ESSAOUIRA - MAROC}

\section{ANDROLOGIE}

\section{Thèmes}

Vieillissement masculin

Infertilité masculine

Physiologie Pharmacologie sexuelle

Prostate en Andrologie

Maladie de La Peyronie

Infection en Andrologie

Communications libres

\section{IMAGERIE GENITO-URINAIRE}

Imagerie prostatique

Imagerie scrotale (hors infertilité)

Imagerie génito-urinaire et infertilité

Imagerie génito-urinaire et pathologie infectieuse

Imagerie pénienne

\section{SEXOLOGIE}

La sexualité dans les cultures méditerranéennes

Faire couple et nouveaux couples

Ages de la femme et de l'homme

Dysfonctions éjaculatoires

Communications libres.

\section{Association Méditerranéenne d'Andrologie}

44, rue Abou Abdellah Naffi, 20100 Maârif, Casablanca Maroc

Tel : (212) 222595 73, Fax : (212) 22986051

E-mail : a.smires@techno.net.ma

http://www.andrologie-maroc.co.ma 


\section{2-5 Octobre 2002 \\ TOURS}

Contact : Pr Pierre Lecomte, Service d'Endocrinologie-Diabétologie CHU Bretonneau, 37044 TOURS CEDEX

Tél : 33 (0)2 474738 06, Fax : 33 (0)2 47473804

e-mail : lecomte@med.univ-tours.fr

\section{CONGRĖS ANGLOPHONES}

\section{5-28 Avril 2002 \\ 27th Annual Meeting of the American Society of Andrology}

Postgraduate Course: 29 avril 2002

Laboratory Workshop: 24 avril 2002

Sheraton Seattle Hotel and Towers in Seattle, Washington, USA

ASA Executive Offices, 74 New Montgomery, Suite 230, San Francisco, CA 94105, USA

Tel: 415-764-4823, Fax: 415-764-4915, E-mail: 105037.1120@ compuserve.com

29 Mai, 1 er Juin 2002

\section{Epididymis III, Third International Conference on the Epididymis \\ Charlottesville, VA, USA}

Barry T. Hinton, Ph.D., Department of Cell Biology, University of Virginia School of Medicine, P.O. Box 800439, Charlottesville, VA 22908

Tel: 804-924-2174, Fax: 804-982-3912, email:bth7c@ virginia.edu

Web site: http://hsc.virginia.edu/epididymis-III

ou

Terry T. Turner, Ph.D., Department of Urology, University of Virginia School of Medicine, P. O. Box 800422, Charlottesville, VA 22908

tel: 804-924-0429, fax: 804-924-8311, email: ttt@virginia.edu

16-20 Juin 2002-01-17

6th Congress of the European Federation of Sexology "Sexuality in a Real and Virtual Environment"

Limassol, Cyprus

ORTRA LTD, 1 Nirim street, 61092 Tel Aviv ISRAEL

Tel: +972 36384444 , Fax: +972 36384455 , E-mail: sexology@ ortra.co.il

26-28 Juin 2002

\section{2nd Mediterranean Congress of Sexual Dysfunction. Amman Jordan}

Dr Mamun Ezzibdeh, Mediterrannean Congress of Sexual Dysfunction, POBox, Amman, Jordan Tel 009626 5411656, E-mail: info@MCSDAmman.com

URL: http://www.MCSDAmman.com 


\section{8th Annual Meeting ESHRE,}

Vienna, Austria

ESHRE Central Office, c/o Bruno Van den Eede, Van Akenstraatt 41, B-1850 Grimbergen, Belgium

Tel +32-2-269-0969, Fax +32-2-269-5600, Email eshre@pophost.eunet.be

\section{Juillet 2002, \\ Annual Meeting of the Society for the Study of Fertility \\ Leeds, UK}

Society for the Study of Fertility, 892A High Street Sawston, Cambridge CB2 4HJ, UK

Tel +44-1223-830665, Fax +44-1223-839804, Email Office@ssf.org.uk

12-18 Aout 2002

\section{6th Asian Congress of Urology - Urology in the IT Era}

Kuala Lumpur, Malaysia

MediTech Media (M) Sdn Bhd, Plaza 138, W 6.08, 138 Jalan Ampang, 50450 Kuala Lumpur, MALAYSIA Tel: +6032162 9098, Fax: +6032162 9078, email: acu2002@meditech.com.my

29-31 Aout 2002

\section{5th Workshop on Carcinoma in situ and Cancer of the Testis}

Copenhagen, Denmark

Svenningsen Workshopsekretariatet, Rigshospitalet, Section 5212

9 Blegdamsvej, DK-2100 Copenhagen, DENMARK

Tel: +45 35455247 ; Fax: +45 35456645 ; e-mail: ses@ $\underline{\text { rh.dk }}$

19-22 Septembre 2002

2nd European Congress of Andrology,

Malmö, Sweden

Malmö Kongressbyr, S:t Gertrud, Östergatan 3, SE-211 25 Malmö, Sweden

Tel: +46 40 258550, Fax: +46 40 258559, Email: info@malmo-congress.com

22-27 Septembre 2002

Symposium on Male Infertility

\& Hands on workshop on Seminology and IUI

In association with International Society for Andrology (ISA)

At Institute of Human Reproduction (IHR), India

Dr. Deepak Goenka, Organising Secretary, Institute of Human Reproduction, Bharalumukh, Guwahati - 781 009, Assam, India

Tel : +91-361-548767, 544560, Fax: +91-361-518195, 521727, Email: dgoenka@ satyam.net.in

12-16 Octobre 2002

\section{Annual Meeting of the American Society for Reproductive Medicine \\ Seattle, Washington, USA}

American Society for Reproductive Medicine, 1209 Montgomery Highway, Birmingham, Alabama, 35216, USA

Tel: 205-978-5000, Fax: 205-978-5005, E-Mail: asrm@asrm.com 


\section{1-25 Octobre 2002-01-17 \\ 11th International Congress on Hormonal Steroids}

Fukuoka, JAPAN,

Congress Secretariat, Dept Medicine and Bioregulatroy Sciences, Graduate School of Medical Sciences, Kyushu University, 3-1-1 Maidashi, Fuuokka, 812-8582 Japan

Tel +81-92-642-5080, Fax +81-92-642-5287/5297

17-21 Octobre 2002

First Asia-Pacific Forum on Andrology

Xiang-Hua Zhou, c/o Editorial Office, Asian Journal of Andrology, 294 Taiyuan Road, Shanghai 200031

Tel: +86216431 1833 ext. 207, Fax: +86216474 2629, e-mail: aja@mail.shcnc.ac.cn

URL: www.AsiaAndro.com

1er - 4 Décembre 2002

5th Congress of the European Society for Sexual and Impotence Research Hamburg, Germany

Prof. Dr. Hartmut Porst

Neur Jungfernstieg $6 a$

D-20354 Hamburg

Tel : +49 40346184 ; Fax : +49 40351117 ; e-mail : Porst20354@aol.com

www.essir-hamburg-2002.de

29 Mars - 1er Avril 2003

28th Annual Meeting of the American Society of Andrology and Postgraduate Course Phoenix, AZ, USA.

Sheraton Crescent Hotel

ASA Office, 2950 Buskirk Avenue, Suite 170

Walnut Creek, CA 94596

Phone: 925/472-5910 ; Fax: 925/472-5901 ; Email: asa@hp-assoc.com

Juillet 2003

Annual Meeting of the Society for the Study of Fertility

Aberdeen, UK

Society@convention.co.jp

URL : http://www2.convention.co.jp/ichs2002

19-22 Juillet 2004

International Congress of Endocrinology

Lisbon, Portugal

Maguelone G Forest,

DRI-INSERM-U.329

Hopital Debrousse, 29 rue Sur Bouvier, 69322 Lyon Cedex 05 France

Tel +33-4-7238-5848; Fax +33-4-7825-6168 ; Email forest@1yon151.inserm/fr 